\title{
$X$-ray transmission calibration of the gate valve for the $X$-ray astronomy satellite XRISM
}

\author{
Takuya Midooka ${ }^{\mathrm{a}, b^{*}}$, Masahiro Tsujimoto ${ }^{\mathrm{a}}$, Shunji Kitamoto ${ }^{\mathrm{c}}$, Nozomi Nakaniwa ${ }^{\mathrm{a}, \mathrm{d}}$, \\ Yoshitomo Maeda $^{a}$, Manabu Ishida ${ }^{a, d}$, Ken Ebisawa ${ }^{a, b}$, Mayu Tominaga ${ }^{a, b}$ \\ a Japan Aerospace Exploration Agency (JAXA), Institute of Space and Astronautical Science (ISAS), 3-1-1 \\ Yoshinodai, Chuo, Sagamihara, Kanagawa, Japan, 252-5210 \\ ${ }^{\mathrm{b}}$ The University of Tokyo, 7-3-1 Hongo, Bunkyo, Tokyo, Japan, 113-8654 \\ ${ }^{\mathrm{c}}$ Rikkyo University, 3-3-4-1 Nishi-ikebukuro, Toyoshima, Tokyo, Japan, 171-8501 \\ ${ }^{\mathrm{d}}$ Tokyo Metropolitan University, 1-1 Minami-Osawa, Hachioji, Tokyo, Japan, 192-0397
}

\begin{abstract}
Resolve onboard the X-ray satellite XRISM is a cryogenic instrument with an X-ray microcalorimeter in a Dewar. A lid partially transparent to X-rays (called gate valve, or GV) is installed at the top of the Dewar along the optical axis. Because observations will be made through the GV for the first few months, the X-ray transmission calibration of the GV is crucial for initial scientific outcomes. We present the results of our ground calibration campaign of the GV, which is composed of a Be window and a stainless steel mesh. For the stainless steel mesh, we measured its transmission using the X-ray beamline at ISAS. For the Be window, we used synchrotron facilities to measure the transmission and modeled the data with (i) photoelectric absorption and incoherent scattering of $\mathrm{Be}$, (ii) photoelectric absorption of contaminants, and (iii) coherent scattering of Be changing at specific energies. We discuss the physical interpretation of the transmission discontinuity caused by the Bragg diffraction in poly-crystal Be, which we incorporated into our transmission phenomenological model. We present the $\mathrm{X}$-ray diffraction measurement on the sample to support our interpretation. The measurements and the constructed model meet the calibration requirements of the GV. We also performed a spectral fitting of the Crab nebula observed with Hitomi SXS and confirmed improvements of the model parameters.
\end{abstract}

Keywords: XRISM, Resolve, Beryllium, gate valve, X-ray transmission, Bragg diffraction.

*Takuya Midooka, midooka@ac.jaxa.jp

\section{Introduction}

The X-ray astronomy satellite XRISM is planned to be launched in the Japan fiscal year $2022 .{ }^{1}$ It is expected to revolutionize high-energy astrophysics with its high-resolution, high-throughput, and non-dispersive X-ray spectrometer based on the X-ray microcalorimetry. The instrument called Resolve is built on the heritage of Hitomi SXS, ${ }^{2}$ which demonstrated its capability in orbit but was discontinued a month after launch due to unexpected failure of the spacecraft attitude control system in $2016 .^{3}$

Resolve is a cryogenic instrument with an X-ray microcalorimeter detector in a Dewar. ${ }^{4,5}$ To maintain the Dewar vacuum on ground and to protect the detector from the initial spacecraft out- 
gassing in orbit, a partially transparent lid called the gate valve (GV) is installed at the top of the Dewar along the X-ray optical path. GV is composed of a Be window and a stainless steel mesh (see details in Section 2). On ground, the GV can be opened and closed repeatedly. In orbit, it can be opened only once using a non-explosive actuator system and cannot be closed once opened. It is planned to be opened during the commissioning phase when the outgassing settles. Until then, all observations will be made through the GV. Therefore, calibrating the energy dependence of the X-ray transmission of this apparatus is crucial for the initial scientific outcomes.

Be windows are routinely used as a standard entrance window in X-ray detectors requiring a leak-tight structure. In the past, the modeling based only on the Be photo-electric absorption was sufficient (e.g. ASCA GIS ${ }^{6}$ ). For X-ray spectrometers with a high spectral resolution and a high throughput such as Hitomi SXS, ${ }^{2}$ XRISM Resolve, ${ }^{4}$ and Athena X-IFU, ${ }^{7}$ this is insufficient. This was recognized in SXS, ${ }^{8}$ based on which we develop the model for Resolve in this work.

In the SXS, the GV was not calibrated using the flight model on ground unlike the other components. ${ }^{9}$ This choice was motivated by the lower priority due to its not being used under nominal observations and some scheduling constraints. Due to the unexpected early loss of the spacecraft, all observations were made with the GV closed. After the loss, the transmission measurements were performed using a spare unit. ${ }^{8-10}$ As a result, the GV turned out to be the cause for the largest systematic uncertainty in the effective area calibration in the Crab observation with the SXS. ${ }^{11}$

Based on this lesson, we planned to perform the ground calibration measurements for all parts of the GV for Resolve with a clear set of calibration requirements that are traced back to science goals of the mission. We aim to understand causes of the large systematic uncertainty in the SXS $\mathrm{GV}$ and reduce it. The allocated budgets for the GV are shown in Table 1. The calibration results will be delivered to users through the calibration database (CALDB). 
Table 1 Calibration requirements of the Be window and the stainless steel mesh ${ }^{12}$

\begin{tabular}{cccccc}
\hline Component & Items & Accuracy & Range (req) & Range (goal) & Comment \\
\hline \hline \multirow{2}{*}{ Be window } & $\begin{array}{c}\text { Broadband } \\
\text { (absolute transmission) }\end{array}$ & $\pm 1 \%$ & $2.5-12.0 \mathrm{keV}$ & $1.8-25.0 \mathrm{keV}$ & \\
& $\begin{array}{c}\text { Fine structures } \\
\text { (relative transmission) }\end{array}$ & $\pm 5 \%$ & & $\begin{array}{c}\text { BDFs and contaminants } \\
\text { Knowledge of structure to 2 eV required }\end{array}$ \\
& $\begin{array}{c}\text { Spatial uniformity } \\
\text { (relative transmission) }\end{array}$ & $\pm 3 \%$ & $\begin{array}{c}\text { single energy } \\
(\sim 3.6 \mathrm{keV})\end{array}$ & With $>100$ spatial grids \\
\hline Mesh & Broadband & $\pm 1 \%$ & $2.5-12.0 \mathrm{keV}$ & $1.8-25.0 \mathrm{keV}$ & \\
\hline
\end{tabular}

We performed a ground calibration campaign using various facilities in 2018-2019 and achieved the required calibration accuracy before the GV was installed in the flight model (FM) of Resolve at the end of 2019. In this paper, we describe the details of this campaign in the following structure. First, we give a brief description of the GV in Section 2. We then present the results of the measurements in Section 3. Based on the results, we construct a physical model of the transmission curve and apply it to the data showing that the best-fit model is consistent with the data within the required calibration accuracy. Finally, in Section 4, we show that we can obtain a better fit to the observed Crab spectrum with the SXS by applying our model with improved parameters. We summarize the results in Section 5. More details can be found in Midooka (2020). ${ }^{13}$

\section{GATE VALVE}

The GV has two parts in the optical path when it is closed (Figure 1): (1) Beryllium window of about $270 \mu \mathrm{m}$ thickness and (2) stainless steel mesh for protecting the Be window from accidental damage by any infalling materials during the ground testing. A picture of these components is shown in Figure 2. They have an inner diameter of $29 \mathrm{~mm}$ and are placed about $231 \mathrm{~mm}$ above the detector. 


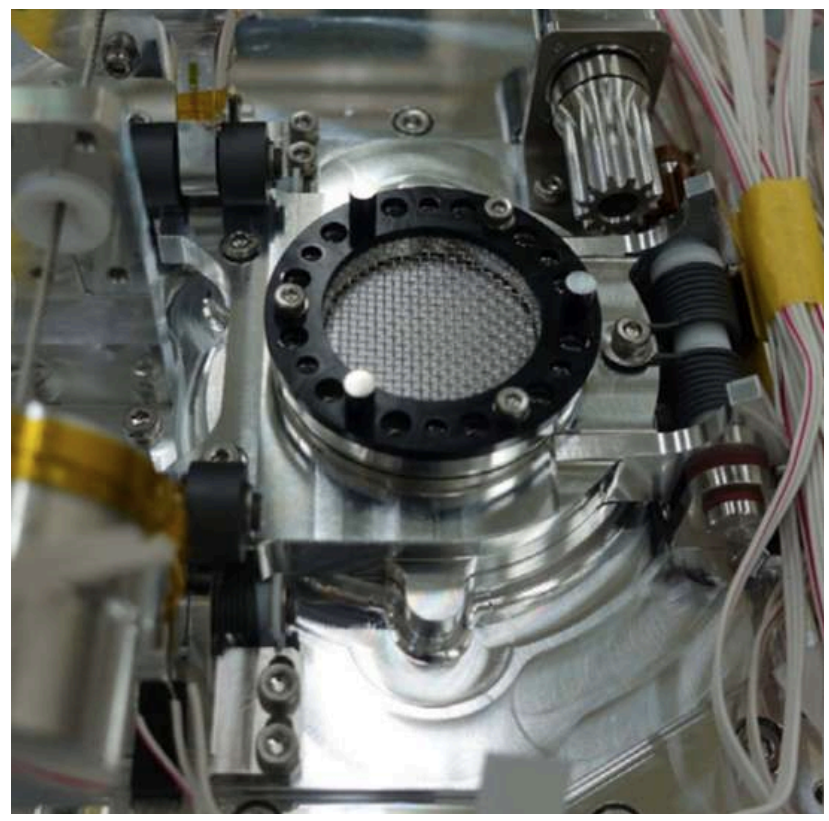

Fig 1 Resolve FM GV in a closed configuration at the top of the Dewar. The photo was provided by Sumitomo Heavy Industries, Ltd.
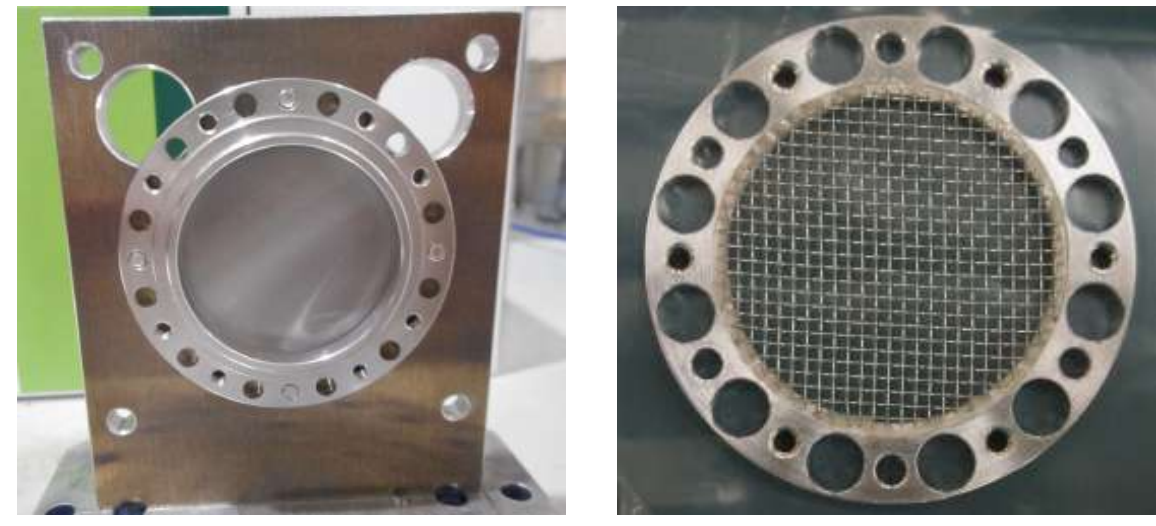

Fig 2 Picture of the Be window (calibration model placed on the holder) and the stainless steel mesh (flight model attached to the flange).

Be window The Beryllium window was fabricated by Materion Corp. from a high purity (IF-1) rolled foil with a reported thickness of $270_{-5}^{+8} \mu \mathrm{m}$. Three models were provided from the same lot, which we use as the flight model (FM), the flight spare (FS), and the calibration model (CM). Although the geometrical thickness was measured by the provider, the X-ray measurements are 
mandatory because (i) the Be window in a thin film form may have a lower density than the values in the literature, (ii) the thickness has some gradient over the diameter in the manufacturing process, and (iii) many spectroscopic features (absorption edges by atoms of impurities and the features caused by Bragg diffraction) may affect the high-resolution science by the microcalorimeter. They are known to vary in different lots from the SXS results. ${ }^{8,10}$

Stainless steel mesh The stainless steel mesh was installed by Sumitomo Heavy Industries, Ltd. The mesh is a plain weave using the SUS 304 stainless steel wires with a circular cross section. In the drawing, the wire thickness is $0.20 \mathrm{~mm}$, the pitch is $1.27 \mathrm{~mm}$, and the wire gap is $1.07 \mathrm{~mm}$. The design values are the same for SXS and Resolve. From these values, the opening fraction is estimated to be $(1.07)^{2} /(1.27)^{2} \sim 71 \%$. The remaining $29 \%$ is opaque to the $\mathrm{X}$-rays except for the highest end of the X-ray bandpass. The mesh for SXS had an optically-thick cross structure at the center for alignment purposes, which was removed for Resolve. The transmission by the presence of this structure is included in the ray-tracing simulation, which is treated separately from the average transmission of the mesh part to be presented in this manuscript. The mesh part was not measured in the SXS.

\section{EXPERIMENTS AND RESULTS}

\subsection{Energy Dependence of the X-ray Transmission of the Stainless Steel Mesh}

We first describe the X-ray transmission measurements of the stainless steel mesh performed using the $30 \mathrm{~m}$ X-ray beamline at JAXA ISAS ${ }^{14}$ from February 4 to 19, 2019. At that time, the FM stainless steel mesh was not manufactured, thus we used a spare mesh left from the SXS. Figure 3 shows the schematic view of the beamline. X-rays from an X-ray generator illuminate one of the 
five metal targets: Ti, $\mathrm{Cu}, \mathrm{Pt}, \mathrm{Mo}$, and $\mathrm{Ag}$. Their fluorescent and scattered X-rays are further monochromatized using a Ge (220) double crystal monochromator (DCM). The stainless steel mesh was installed on the sample stage. The parallel beam was formed by a slit of $2.0 \times 2.0 \mathrm{~mm}$, which is comparable in size with the mesh pitch.

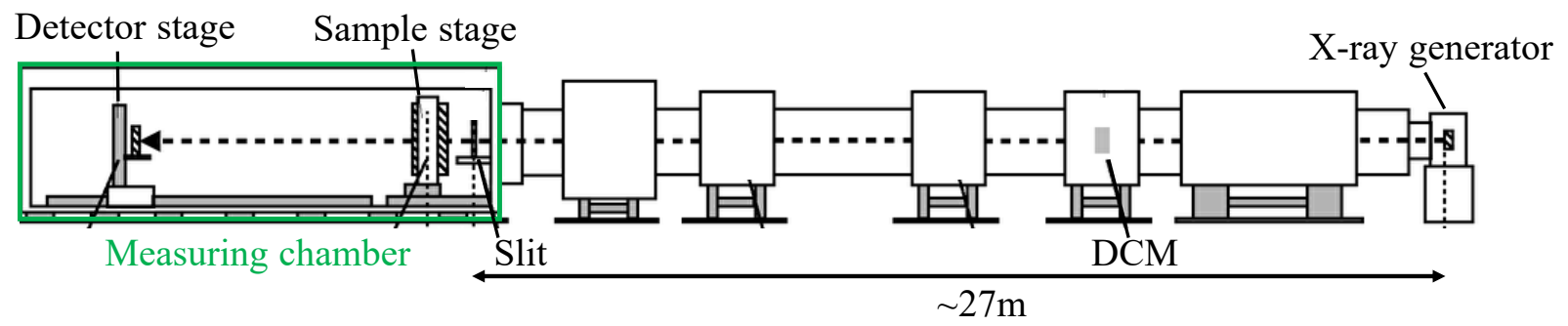

Fig 3 Schematic of the ISAS beamline. ${ }^{14}$ The distance between X-ray generator and a slit is approximately $27 \mathrm{~m}$. The slit, sample stage, and detector stage are installed in the measuring chamber.

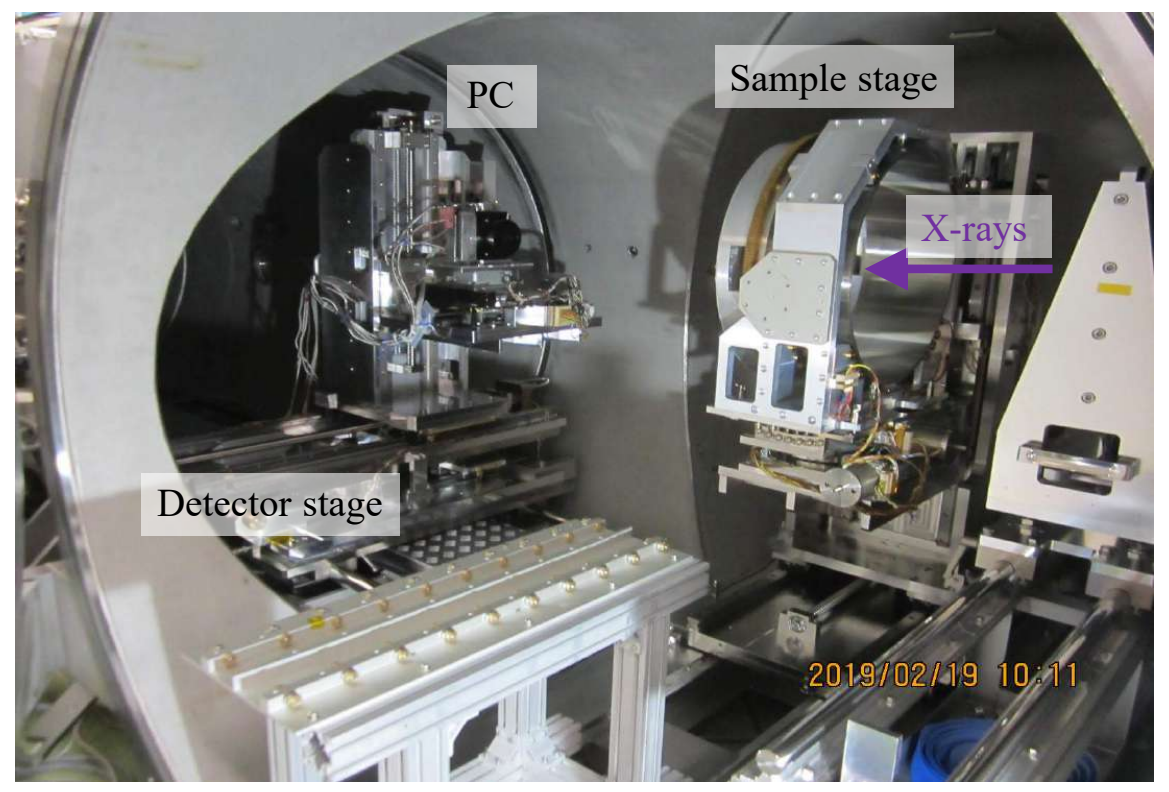

Fig 4 Interior of the measuring chamber in the beamline.

X-ray spectrum with and without the mesh on the sample stage was measured with the proportional counter (PC) filled with a P10 gas (Ar: 90\%, $\left.\mathrm{CH}_{4}: 10 \%\right)$ on the detector stage. We call them transmitted and direct X-rays, respectively. For the transmitted X-rays, we performed raster 
scans over the entire mesh to average for the mesh pattern. A raster scan is composed of several zigzag lines over entire surface of the mesh by moving the sample stage continuously at a slow speed of $0.1 \mathrm{~mm} \mathrm{~s}^{-1}$. The entire surface is scanned many times to make the systematic error small enough. We performed direct X-ray measurements before and after the transmitted X-ray measurements without moving the stages. This set was repeated several times to accumulate sufficient photon statistics. We then calculated the transmission of the mesh by taking the ratio between the transmitted and direct X-ray photons in an energy band of interest for each target line. The result is shown in Table 2 and the red points with errors in Figure 9. Measurement errors include systematic and statistical errors, though the latter is negligible. The former was estimated as variations among individual scans and the latter as Poissonian of the photon counts.

Table 2 Transmission measurement results of the stainless steel mesh

\begin{tabular}{|c|c|c|c|c|c|c|}
\hline Target & $\begin{array}{c}\text { Energy } \\
{[\mathrm{keV}]}\end{array}$ & Sample $^{\mathrm{a}}$ & $\begin{array}{c}\text { Total exp. } \\
\text { [sec] }\end{array}$ & $\begin{array}{c}\text { Total photons } \\
\text { [counts] }\end{array}$ & $\begin{array}{c}\text { Transmission } \\
{[\%]}\end{array}$ & $\begin{array}{c}\text { Error }^{\mathrm{b}} \\
{[\%]}\end{array}$ \\
\hline \multirow{2}{*}{$\mathrm{Ti} \mathrm{K} \alpha$} & \multirow{2}{*}{4.51} & ON & 1866.3 & 2562596 & \multirow{2}{*}{72.7} & \multirow{2}{*}{ \pm 0.6} \\
\hline & & OFF & 3627.8 & 6850815 & & \\
\hline \multirow{2}{*}{$\mathrm{Cu} \mathrm{K} \alpha$} & \multirow{2}{*}{8.05} & ON & 1856.7 & 200030 & \multirow{2}{*}{71.7} & \multirow{2}{*}{ \pm 0.9} \\
\hline & & OFF & 3601.7 & 540877 & & \\
\hline \multirow{2}{*}{$\operatorname{Pt} \mathrm{L} \beta$} & \multirow{2}{*}{11.07} & ON & 1858.0 & 422722 & \multirow{2}{*}{71.5} & \multirow{2}{*}{ \pm 1.5} \\
\hline & & OFF & 3605.6 & 1149485 & & \\
\hline \multirow{2}{*}{ Mo K $\alpha$} & \multirow{2}{*}{17.48} & ON & 3712.8 & 211733 & \multirow{2}{*}{72.3} & \multirow{2}{*}{ \pm 2.3} \\
\hline & & OFF & 3601.6 & 285442 & & \\
\hline \multirow{2}{*}{ Mo $\mathrm{K} \beta$} & \multirow{2}{*}{19.61} & ON & 11136.2 & 122811 & \multirow{2}{*}{73.6} & \multirow{2}{*}{ \pm 2.8} \\
\hline & & OFF & 7200.1 & 109824 & & \\
\hline \multirow{2}{*}{$\operatorname{Ag~K} \alpha$} & \multirow{2}{*}{22.17} & ON & 1861.2 & 270694 & \multirow{2}{*}{76.4} & \multirow{2}{*}{ \pm 1.3} \\
\hline & & OFF & 3613.6 & 686769 & & \\
\hline
\end{tabular}

${ }^{a}$ Sample ON/OFF means the measurements of the transmitted/direct X-rays.

b The error is for a $1 \sigma$ level.

\subsection{Energy Dependence of the X-ray Transmission of the Be Window}

X-ray transmission measurements of the FM Be window were performed at KEK Photon Factory (PF) from June 13 to 23,2019 . We used the beamlines BL-7C ${ }^{15}$ and BL-11B. ${ }^{16}$ The operating 
energy ranges are 4.0-12.0 keV and $2.1-5.0 \mathrm{keV}$ and we used a beam size of $1.0 \times 1.0 \mathrm{~mm}$ and $2.5 \times 2.5 \mathrm{~mm}$ as the largest possible square respectively for BL-7C and BL-11B. The intense synchrotron X-rays are monochromatized using a Si (111) DCM. We measured three positions of the Be window; they are at the center of the window and at two positions $6.5 \mathrm{~mm}$ off-center (top and bottom).

Figure 5 shows the schematic drawings of the setup for measurements at the BL-11B and BL-7C. ${ }^{8}$ In the BL-11B experiments, we attached our vacuum chamber to the beamline through a Kapton polyimide film and installed the Be window and a photo diode inside the chamber to prevent absorption by the air as shown in Figure 6. The incident X-rays were measured using a Ti foil as a photocathode beam monitor provided by the facility. In the BL-7C experiments, we measured incident and transmitted X-rays by two ion chambers filled with $\mathrm{N}_{2}$ gas provided by the facility in front and back of the Be window placed in the air.

The contamination by higher-order X-rays are removed by a focusing double mirror in the BL-7C measurements. The fraction of the remaining third-order $\mathrm{X}$-ray contamination at $4 \mathrm{keV}$ is negligible of about $3 \times 10^{-5} \cdot{ }^{15}$ The contamination has not been reported in the BL-11B in past experiments at least above $2.3 \mathrm{keV}$, hence we consider that they are negligible at the higher energy range in our measurements. 

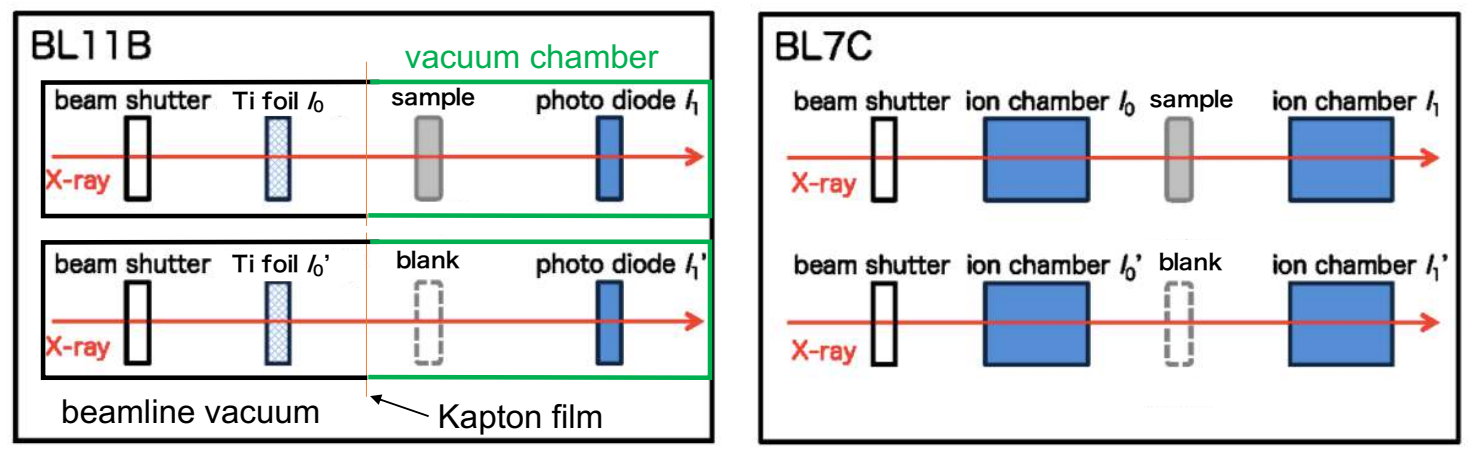

Fig 5 Schematic drawings of the setup for measurements at the BL-11B and BL-7C. ${ }^{8}$

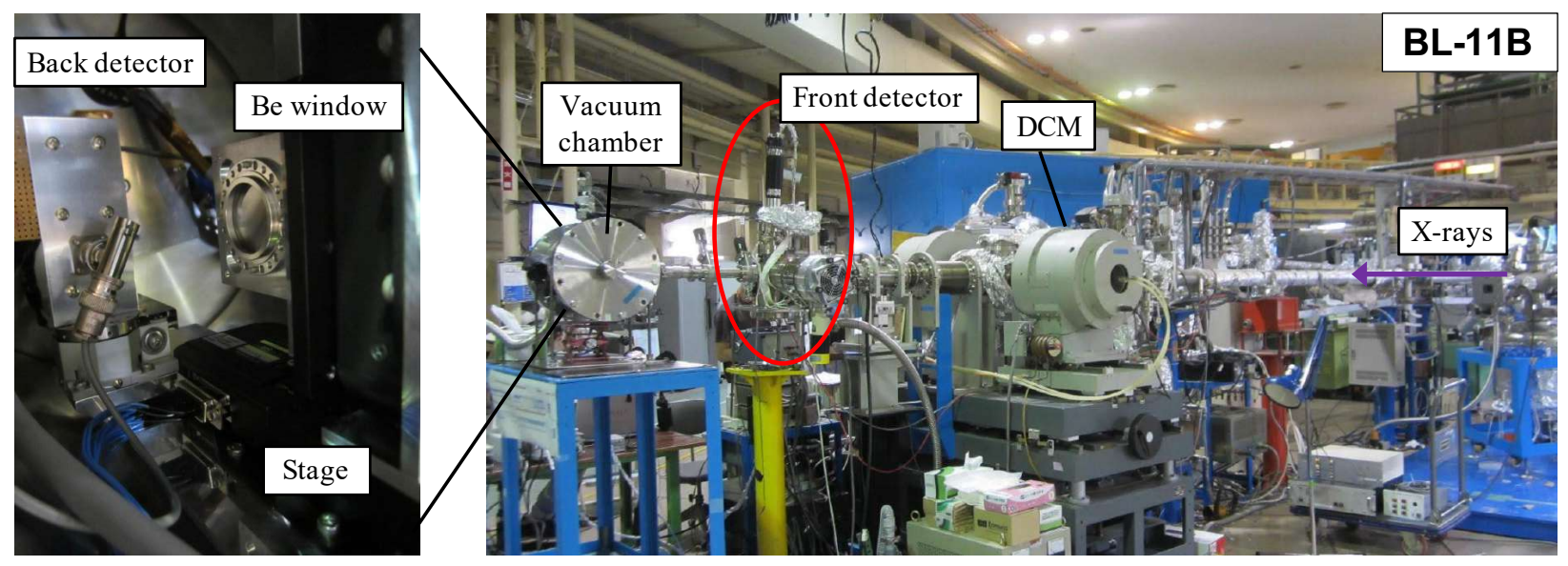

Fig 6 right: The photo of the BL-11B. left: Interior of the vacuum chamber. Be window and a photo diode as the back-side detector are installed.

In both experiments, we measured current of the detectors (i.e. the ion chambers, the photodiode, and the Ti foil), as the intensity of the incident and transmitted X-rays. Here, we assume that $I_{0}(E)$ and $I_{1}(E)$ are the incident and transmitted X-ray intensities with the Be window, and $I_{0}^{\prime}(E)$ and $I_{1}^{\prime}(E)$ are those without the Be window at a given energy $E$. The dark current was measured and subtracted from them. We derived the transmission by

$$
T(E)=\frac{I_{1}(E) / I_{0}(E)}{I_{1}^{\prime}(E) / I_{0}^{\prime}(E)}
$$


With this double normalization, we can correct for any instability of the beam as well as the energy dependence caused by the measurement setup. We repeated this measurement with a step of $2 \mathrm{eV}$ in eight energy ranges overlapping with each other at 2.35-2.6, 2.55-3.0, 2.95-3.5, 3.45-4.0 keV bands in BL-11B, and 4.0-5.0, 4.9-8.0, 7.9-10.0, 9.9-12.0 keV bands in BL7C. The overlapped range is 50 or $100 \mathrm{eV}$.

The upper panels of Figure 7 show derived transmission curves of the top position of the Be window and model curves in $2.1-4.5 \mathrm{keV}$ at the BL-11B (left) and in 4.0-12.0 keV at the BL-7C (right). The ratio between two adjacent energy ranges was corrected by multiplying a constant to the data set in the lower energy of the two. This was repeated to construct a continuous transmission curve over the entire energy. The seven constant values provide an estimate of the systematic uncertainty in the measurements. The mean and the standard deviation of them are 1.00000 and 0.00081, respectively. We thus evaluate the systematic uncertainty of the measurements to be $0.08 \%$.
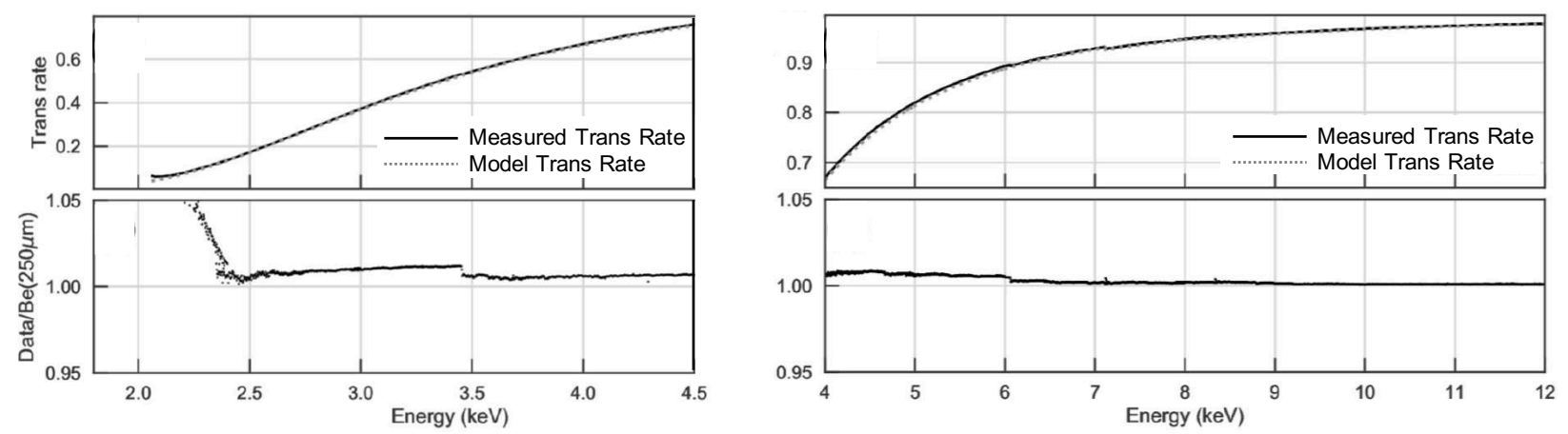

Fig 7 Upper panel: A model transmission curve assuming a photoelectric absorption by Be of a $250 \mu \mathrm{m}$ thickness and the transmission measured at the top position of the Be window obtained at the BL-11B (left) and BL-7C (right). Lower panel: The ratio of the measurement curve to the model curve. The anomalous increase of the transmission below $2.4 \mathrm{keV}$ is attributable to higher-order X-ray reflections in the DCM.

The lower panels of Figure 7 show the ratio of the measurement against the model assuming photoelectric absorption by Be of a $250 \mu \mathrm{m}$ thickness. Here, we used the thickness only for dis- 
playing purpose to show the residual structures in a limited range. We found an apparent increase in the transmission below $2.4 \mathrm{keV}$. After the delivery of FM Be window, we made an additional measurement using another model (CM) to investigate this issue at the beamline BL-11 of the Hiroshima Synchrotron Radiation Center (HiSOR) ${ }^{17}$ in October 2019. In HiSOR, we examined the contribution of higher-order X-rays by detuning the two crystal angles in the DCM. The result suggested some influence of higher-order X-rays in the measurements at KEK PF. We conclude that the anomalous increase of the transmission below $2.4 \mathrm{keV}$ (Figure. 7 ) is likely caused by the effect of higher-order X-ray reflections ${ }^{13}$ and decided to ignore the data below $2.6 \mathrm{keV}$ in the following analysis.

\subsection{Spatial Dependence of the X-ray Transmission of the Be Window}

We evaluated the spatial dependence of the transmission using the FM Be window. We used the beamline BL-11B using almost the same setup in Section 3.2. The beam size is adjusted to $0.8 \mathrm{~mm}$ (horizontal) $\times 1.2 \mathrm{~mm}$ (vertical). We measured the X-ray transmission at some fixed energy over the entire Be window by a scan. We chose four energies: 2700, 3000, 3456, and $4000 \mathrm{eV}$. The scan was continuous in the horizontal direction at a speed of $500 \mu \mathrm{m} \mathrm{s}^{-1}$ and step-wise in the vertical direction with a step size of $1 \mathrm{~mm}$. The time series in the horizontal scan was converted to the positions by the speed and rebinned into a grid map. At the beginning and at the end of each horizontal scan, we obtained reference transmission without the Be window for $10 \mathrm{~s}$.

Figure 8 shows the 2D maps of the transmission of the FM Be window. In all energies, transmission at the lower left of the Be window is higher than the average value. We speculate that this is caused in the manufacturing process. In the $4000 \mathrm{eV}$ map, the mean and $3 \sigma$ scatter of the transmission is $66.8_{-1.1}^{+1.0} \%$, which translates to a thickness of $251.5 \pm 10.5 \mu \mathrm{m}$. The non-uniformity 
is $\pm 4.2 \%$ at $3 \sigma$.
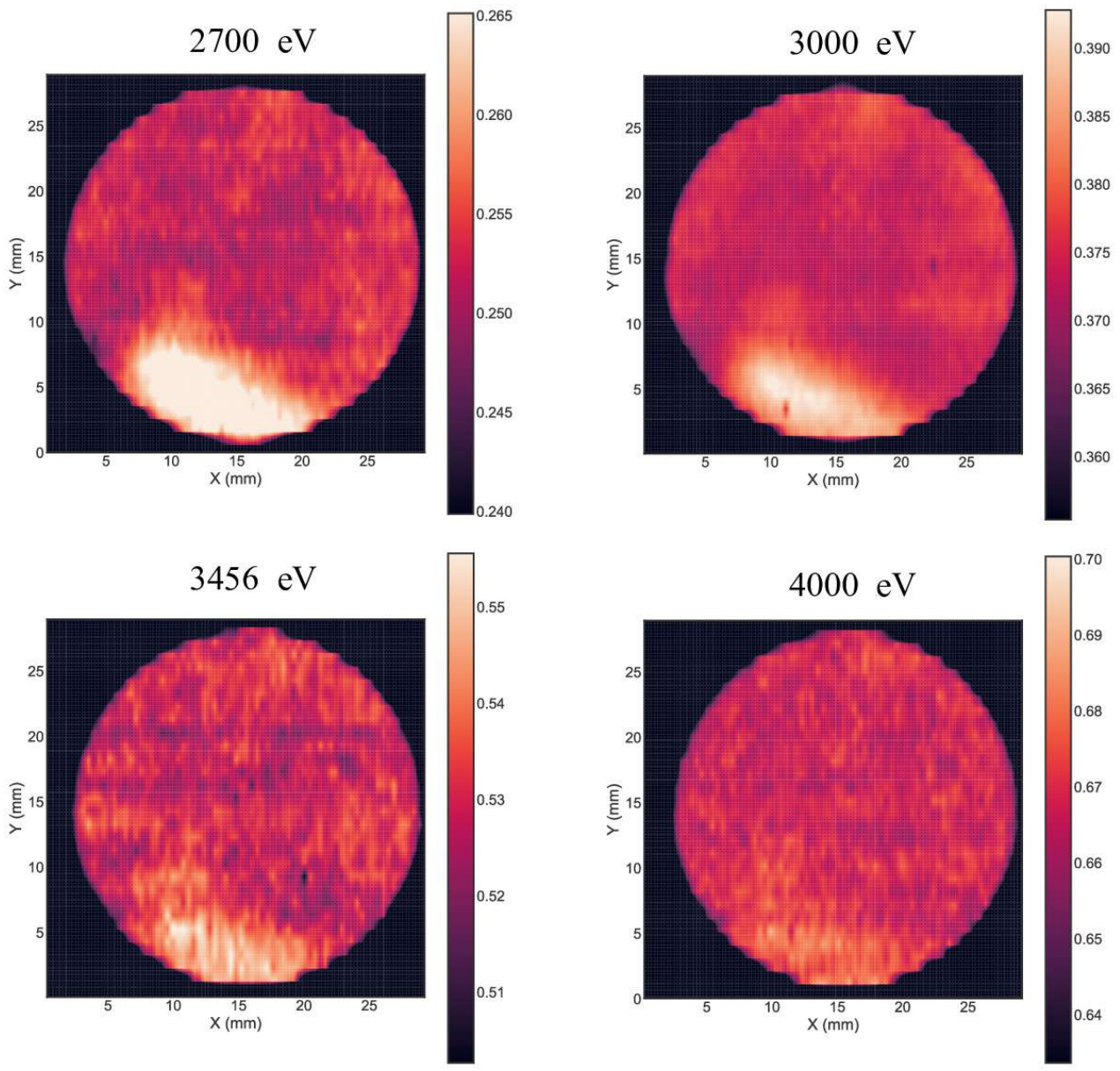

Fig 8 Spatial non-uniformity maps of the transmission of the FM Be window at four selected energies. The figures are aligned to the spacecraft coordinate: i.e, $+\mathrm{X},+\mathrm{Y}$ directions in the figure are aligned to $\mathrm{SAT}+\mathrm{X}$ and $\mathrm{SAT}+\mathrm{Y}$. These maps were faced in the direction viewed from the incident X-rays both in the experiment and also to the in-orbit setup. The fuzzy boundary is due to an artifact as a result of circular masking.

\section{DISCUSSION}

\subsection{Modeling Transmission of the Stainless Steel Mesh}

In Figure 9, the measured transmission (Table 2) is shown with the red points with errors, whereas the model curve adopted in the SXS CALDB is with the blue curve. The mesh is made of SUS 304, which we assumed to have $70 \% \mathrm{Fe}, 20 \% \mathrm{Cr}$, and $10 \% \mathrm{Ni}$. The transmission is modeled by a product of the photoelectric absorption and scattering attenuation of these elements. 
The model curve adopted in the SXS was found inconsistent with the measurements, in particular, for the low energy end in Ti $\mathrm{K} \alpha(4.51 \mathrm{keV})$ and the high energy end in $\mathrm{Ag} \mathrm{K} \alpha(22.17 \mathrm{keV})$. One reason is that the cross section of the mesh wire was assumed a square with $0.2 \mathrm{~mm} \times 0.2 \mathrm{~mm}$ in SXS, while it is round in reality, hence the actual average depth is smaller than $0.2 \mathrm{~mm}$. The transmission is non-linear to the depth, so we performed numerical integration assuming that the wire depth is $2 \sqrt{\left(t^{2} / 4-x^{2}\right)}$ at $x(<t / 2)$ away from the center of the wire with a diameter of $t=0.2 \mathrm{~mm}$. Using the mass attenuation coefficient of a constituent metal $\mu_{\mathrm{tot}, \mathrm{metal}}(E)$, we derived the total attenuation coefficient $\alpha(E)$ as

$$
\alpha(E)=\sum_{\text {metal }} A_{\text {metal }} * \rho_{\text {metal }} * \mu_{\text {tot, } \text { metal }}(E),
$$

where $A_{\text {metal }}$ is the mass fraction and $\rho_{\text {metal }}$ is the mass density of the constituents $(\mathrm{Fe}, \mathrm{Cr}, \mathrm{Ni})$. The transmission of the stainless steel mesh $T_{\text {mesh }}(E)$ is then calculated as:

$$
T_{\text {mesh }}(E)=f+(1-f) \int_{0}^{\frac{t}{2}} \exp \left(-2 \sqrt{t^{2} / 4-x^{2}} * \alpha(E)\right) d x
$$

where $f$ is the opening fraction of the mesh. We note that the contribution of the overlapped parts of the mesh wires can be ignored compared to the measurement error in Table 2.

First, we fixed $f$ to 0.71 estimated based on the design in Section 2, which is shown with the orange curve in Figure 9. This is still inconsistent with the measurements. Considering some uncertainty in the estimate of $f$, we treated it as a free parameter. The best-fit value was $0.723 \pm 0.003$. The resultant model is shown with the green curve in Figure 9, which is now consistent with the measurements. We adopt this model for Resolve. 


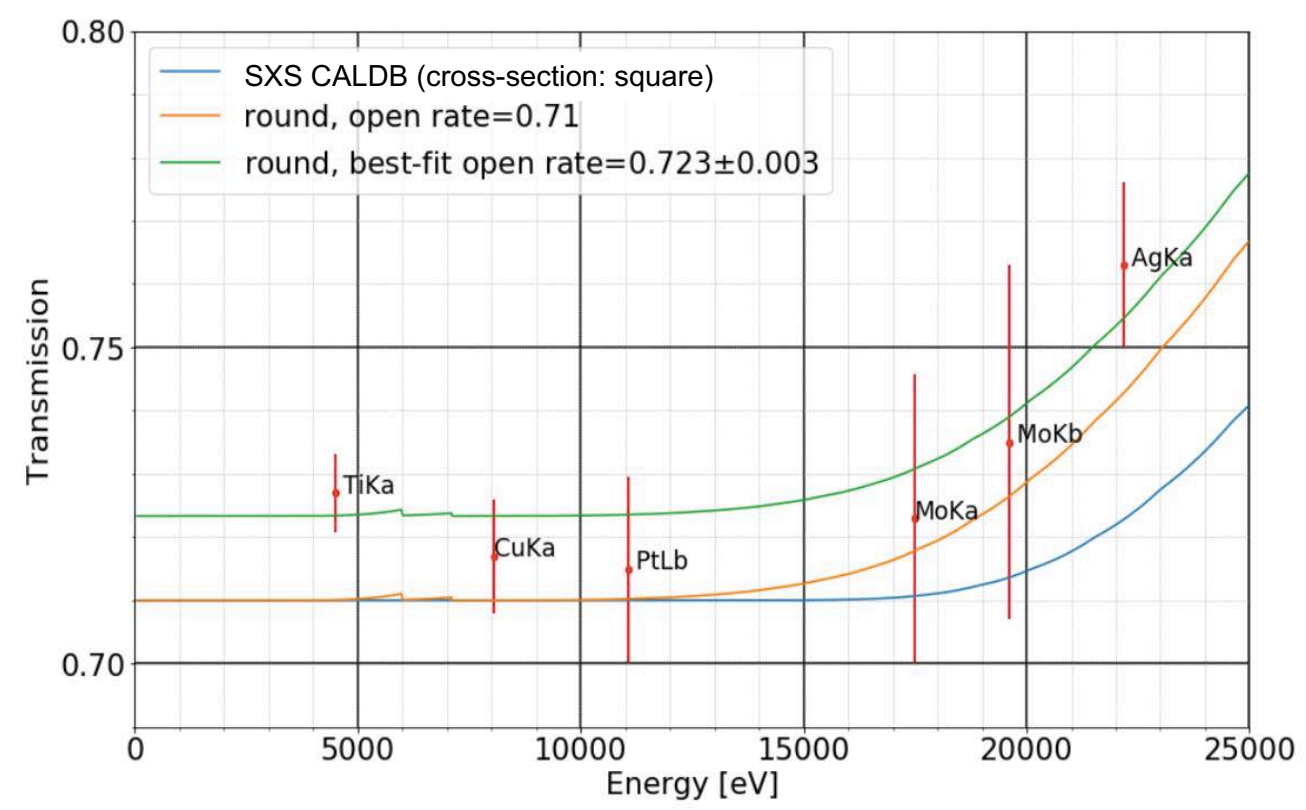

Fig 9 Comparison of three transmission models for the stainless steel mesh. Red points describe the measurement results with errors. Blue, orange, green curves show the SXS CALDB model, a round cross-section model with a fixed $71 \%$ opening fraction, and the Resolve model.

\subsection{Modeling Transmission of the Be Window}

First, we investigated contamination by impurities. In the lower panel of Figure 7, we identified features associated with the photoelectric absorption edges of $\mathrm{Cr}-\mathrm{K}, \mathrm{Mn}-\mathrm{K}, \mathrm{Fe}-\mathrm{K}, \mathrm{Ni}-\mathrm{K}$, and $\mathrm{Cu}-\mathrm{K}$, but not others. We derived their areal densities by local fitting and summarize the result in Table 3. The result is compared with the report by Materion Corp.

Table 3 Best-fit areal density of the impurities based on the transmission measurements of the FM Be window.

\begin{tabular}{ccccc}
\hline $\begin{array}{c}\text { Impurity } \\
\text { element }\end{array}$ & $\begin{array}{c}\text { Edge energy } \\
{[\mathrm{eV}]}\end{array}$ & $\begin{array}{c}\text { Fitting energy } \\
\text { range }[\mathrm{eV}]\end{array}$ & $\begin{array}{c}\text { Best-fit areal }^{\mathrm{a}} \\
\text { density }\left[\mu \mathrm{g} / \mathrm{cm}^{2}\right]\end{array}$ & $\begin{array}{c}\text { Reference } \mathrm{areal}^{\mathrm{b}} \\
\text { density }\left[\mu \mathrm{\mu g} / \mathrm{cm}^{2}\right]\end{array}$ \\
\hline $\mathrm{Cr}$ & 5989 & $5500-6050$ & $0.8 \pm 0.1$ & 1 \\
$\mathrm{Mn}$ & 6539 & $6510-6900$ & $<0.2$ & 1 \\
$\mathrm{Fe}$ & 7112 & $7000-7500$ & $11.4 \pm 0.2$ & 9 \\
$\mathrm{Ni}$ & 8333 & $8200-8450$ & $7.7 \pm 0.2$ & 5 \\
$\mathrm{Cu}$ & 8979 & $8800-9180$ & $0.7 \pm 0.1$ & 1 \\
\hline
\end{tabular}

a The uncertainties are at the $1 \sigma$ confidence level.

$\mathrm{b}$ The values are derived from the fraction of materials measurements by Materion Corp. 
We also detected other edge-like features at $3460,6057 \mathrm{eV}$, and so on. They are physically interpreted as the Bragg diffraction in the Be window. ${ }^{8} 10$ Here, the Be window is a poly-crystal, in which a part of the material satisfies the Bragg condition at any given energy. An ideal case is called the powder diffraction and the diffracted X-rays form a ring called the Debye-Scherrer ring. Hereafter, we call these edge-like features as Bragg diffraction features (BDFs). When the incident X-ray energy satisfies the Bragg condition expressed in Equation (4) for the lattice spacing $d_{h k l}$ of the Miller index $(h k l)$, X-rays are coherently scattered into a Debye-Scherrer ring. The ring disappears when the incident energy becomes lower than the BDF energy $E_{h k l}(\theta=\pi / 2)$, as the scattered angle cannot exceed $2 \theta=180^{\circ}$. This makes a discontinuity in the measured transmission curve at $E_{h k l}(\theta=\pi / 2)$ of all Miller indices except for those with systematic absence (Appendix A).

$$
E_{h k l}(\theta)=\frac{h c}{2 d_{h k l} \sin \theta},
$$

where $\theta$ is the diffraction angle, $h$ is the Planck constant, and $c$ is the speed of light.

The interpretation was confirmed in an additional measurement using CM. We performed an Xray diffraction measurement using a multi-purpose X-ray diffractometer (Rigaku Ultima IV) in the Tokyo Metropolitan University. Figure 10 shows the intensity of the scattered $\mathrm{Cu} \mathrm{K} \alpha$ line X-rays as a function of the diffraction angle. All peaks are identified with a Miller index and correspond to a Debye-Scherrer ring. The peak heights in the diffraction is in an agreement with the edge depths in the transmission for each Miller index. The result endorses our interpretation that the features in the transmission curve are indeed the BDFs. 


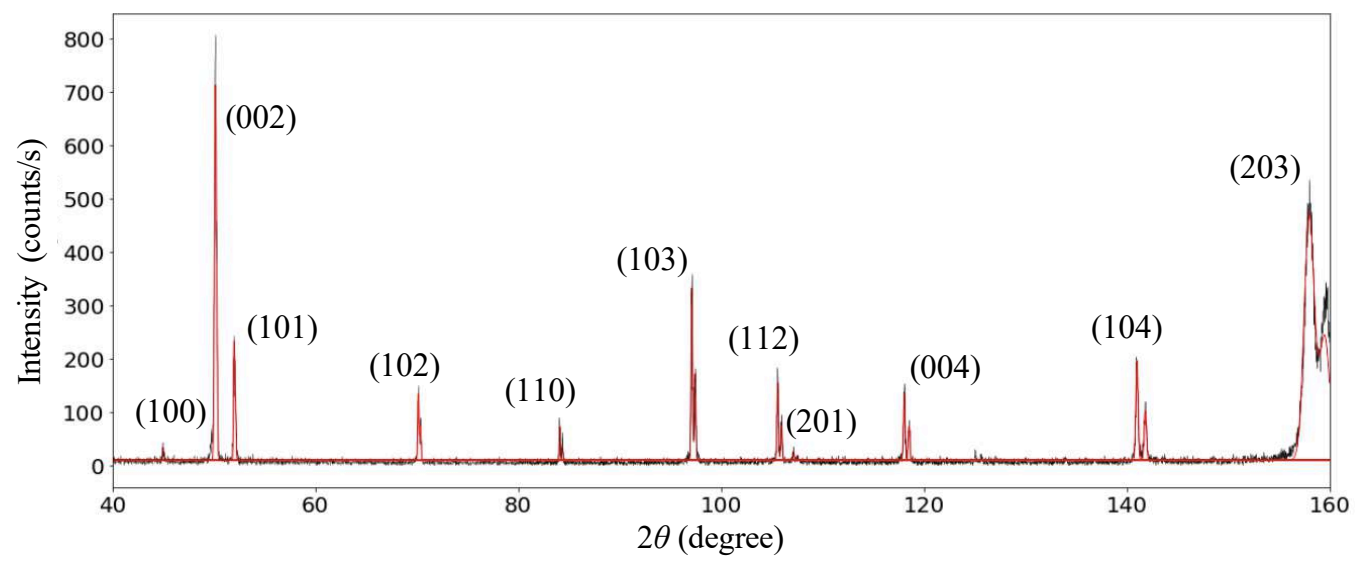

Fig 10 Results of the X-ray diffraction measurements and fitting. All peaks are identified with a Miller index at an expected diffraction angle. All peaks have double-peak structures due to mixing of the $\mathrm{Cu} \mathrm{K} \alpha^{1}$ and $\mathrm{K} \alpha^{2}$ lines in the incident X-rays.

We took the following approach in modeling the BDFs. We used the thickness of the photoelectric absorption, coherent scattering, and incoherent scattering as phenomenological fitting parameters. The energy dependence of the mass attenuation coefficients for the three interactions was fixed to the values by the NIST database. ${ }^{18}$ This approach was motivated by the fact that the scattering cross section effectively changes in lattice from the values in atoms in dynamical diffraction theories. ${ }^{19}$ Considering that the BDF is caused by the energy dependence of the anisotropy of coherent scattering, we changed the coherent scattering thickness in a step-wise manner at all BDFs. In between two BDFs, we assumed that the thickness is proportional to the inverse square of the incident energy (Figure 11). This is derived from the discussion ${ }^{20}$ on the cross section of the powder diffraction as

$$
\frac{d \sigma}{d \Omega}=N_{c}\left(\frac{d_{\tau}^{3}}{v_{0}}\right) M_{\tau}|F(\tau)|^{2} \frac{\tan \theta}{2 \pi} \delta(\gamma)
$$

in which $N_{c}$ is the number of unit cells in the powder crystal, $v_{0}$ is the volume of a unit cell, $\tau$ is the reciprocal vector corresponding to a Miller index, $F(\tau)$ and $M_{\tau}$ are respectively the structure 
factor and the reflection multiplexity factor for the reflection $\tau$, and $d_{\tau}$ is the lattice spacing. The Bragg condition is met at $h c / E=2 d_{\tau} \sin \theta$. The angle $\gamma=2 \theta$ and $d \Omega=2 \pi \sin \gamma d \gamma$. The Equation (5) is derived for the constant-wave case, but it applies to the constant-energy case as well as wavelength-dispersive case with variable conversions. The scattered photon counts per second integrated over the entire solid angle (or entire energy range through the Bragg condition) is then given by

$$
P_{\tau}(E)=\int d E^{\prime} \frac{d \sigma}{d \Omega} \frac{d \Omega}{d E^{\prime}} \Phi\left(E^{\prime}\right)=N_{c}\left(\frac{d_{\tau}}{v_{0}}\right) M_{\tau}|F(\tau)|^{2} \Phi(E) \frac{h^{2} c^{2}}{4 E^{2}}
$$

in which $\Phi(E)$ is the incident photon counts per second per unit area per energy bin. From these, $P_{\tau}(E) / \Phi(E) \propto E^{-2}$, which translates to the thickness dependence on $E$ when the scattering depth is optically thin. The thickness was left constant against energy beyond $\sim 9.2 \mathrm{keV}$, where there are numerous BDFs and we observed no discontinuous structures in the data.

Table 4 shows best-fit coherent scattering thickness of the Resolve FM Be window, which accounts for BDFs. Considering these values and the dependency of $E^{-2}$, we made the plot of the blue curve in Figure 11. 
Table 4 Fitting results of the coherent scattering thickness at each BDF for the Resolve FM Be window.

\begin{tabular}{|c|c|c|c|c|}
\hline $\begin{array}{l}\text { Miller index } \\
\quad h k l\end{array}$ & $\begin{array}{l}\text { BDF energy } \\
{[\mathrm{keV}]}\end{array}$ & $\begin{array}{c}\text { Fitting range } \\
{[\mathrm{keV}]}\end{array}$ & $\begin{array}{c}\text { Coherent scattering } \\
\text { thickness } t_{\mathrm{Be}, \mathrm{coh}}[\mu \mathrm{m}]\end{array}$ & $\begin{array}{c}t_{\mathrm{Be}, \mathrm{coh}} \text { difference }{ }^{\mathrm{b}} \\
\text { above/below BDF energy }[\mu \mathrm{m}]\end{array}$ \\
\hline 100 & 3.1326 & N/A & $0^{\mathrm{a}}$ & 0 \\
\hline 002 & 3.4596 & $3.47-3.57$ & $115.2 \pm 1.6$ & 115.2 \\
\hline 101 & 3.5785 & $3.59-4.66$ & $150.5 \pm 0.8$ & 42.8 \\
\hline 102 & 4.6671 & $4.67-5.42$ & $123.5 \pm 0.8$ & 35.0 \\
\hline 110 & 5.4259 & $5.43-6.05$ & $88.5 \pm 0.7$ & 0.02 \\
\hline 103 & 6.0616 & $6.07-6.25$ & $158.2 \pm 1.1$ & 85.0 \\
\hline 200 & 6.2653 & $6.27-6.43$ & $156.0 \pm 0.9$ & 7.9 \\
\hline 112 & 6.4350 & $6.44-6.49$ & $159.4 \pm 1.4$ & 11.3 \\
\hline 201 & 6.4999 & $6.51-6.91$ & $167.9 \pm 0.6$ & 11.9 \\
\hline 004 & 6.9191 & $6.93-7.1$ & $171.2 \pm 0.6$ & 23.0 \\
\hline 202 & 7.1569 & $7.16-7.59$ & $178.0 \pm 0.5$ & 18.1 \\
\hline 104 & 7.5953 & $7.6-8.13$ & $172.7 \pm 0.4$ & 14.6 \\
\hline 203 & 8.1353 & $8.14-8.78$ & $178.6 \pm 0.9$ & 28.1 \\
\hline 114 & 8.7929 & $8.8-9.19$ & $192.9 \pm 0.5$ & 40.1 \\
\hline 105 & 9.1988 & $9.2-12.0$ & $208.0 \pm 0.2$ & 31.7 \\
\hline
\end{tabular}

${ }^{\mathrm{a}}$ We set $t_{\mathrm{Be}, \mathrm{coh}}=0$ below the lowest BDF energy at the (002) plane.

$\mathrm{b}$ These values are used to plot blue curve in Figure 11.

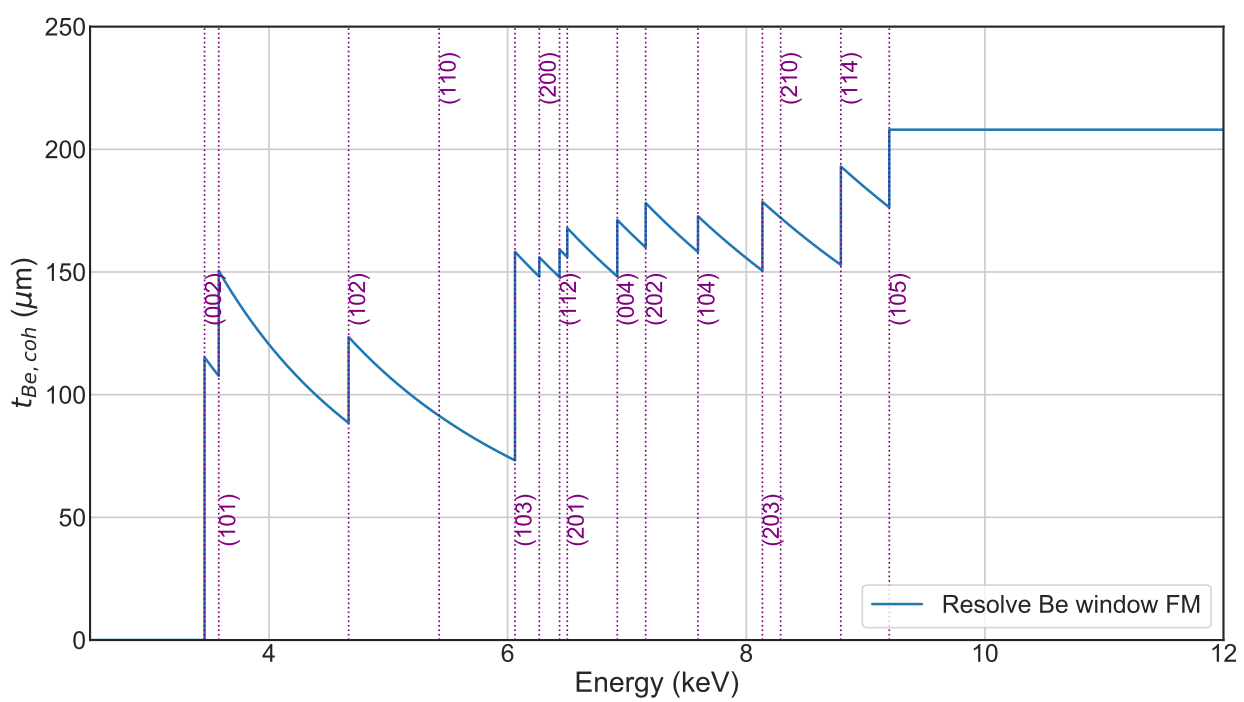

Fig 11 Best-fit thickness of coherent scattering of Be with step-wise changes. Purple lines represent the BDF energies apparent in the data, and the numbers in parentheses represent the crystal planes $(h k l)$ that cause the BDF. We summarize the actual $t_{\mathrm{Be}, \mathrm{coh}}(E)$ values in Table 4 .

Our model consists of the following components: 
- Photoelectric absorption and incoherent scattering by the bulk Be to account for the overall shape of the transmission curve.

- Photoelectric absorption by impurities ( $\mathrm{Cr}, \mathrm{Mn}, \mathrm{Fe}, \mathrm{Ni}$, and $\mathrm{Cu}$ ) to account for the absorption edges.

- Coherent scattering with step-wise changes in thickness to account for the BDFs of Be polycrystals.

The total transmission is given by

$$
T_{\mathrm{Be}}(E)=T_{\mathrm{Be}, \text { photo+inc }}(E) * T_{\mathrm{imp}, \text { photo }}(E) * T_{\mathrm{BDF}}(E),
$$

where $T_{\mathrm{Be} \text {,photo+inc }}(E)$ is the transmission due to the photoelectric absorption and incoherent scattering by the bulk Be expressed as:

$$
T_{\mathrm{Be}, \text { photo }+ \text { inc }}(E)=\exp \left\{-t_{\mathrm{Be}, \text { photo }+ \text { inc }} * \rho_{\mathrm{Be}} *\left(\mu_{\mathrm{Be}, \text { photo }}(E)+\mu_{\mathrm{Be}, \text { inc }}(E)\right)\right\} .
$$

Here, $t_{\mathrm{Be} \text {,photo+inc }}$ is the thickness of the bulk Be common to the photoelectric absorption and the incoherent scattering. $\rho_{\mathrm{Be}}$ is the Be density. $\mu_{\mathrm{Be}, \text { photo }}(E)$ and $\mu_{\mathrm{Be}, \text { inc }}(E)$ are, respectively, the mass attenuation coefficient of photoelectric absorption and incoherent scattering as a function of incident X-ray energy $E$. $T_{\mathrm{imp} \text {,photo }}(E)$ is the transmission due to the photoelectric absorption by the impurities, which is calculated by

$$
T_{\text {imp,photo }}(E)=\prod_{\text {imp }} \exp \left\{-\sigma_{\text {imp }} * \mu_{\text {imp,photo }}(E)\right\}
$$


where $\sigma_{\text {imp }}$ is the areal density of the impurities present in the bulk Be. We modeled the transmission of the BDFs by

$$
T_{\mathrm{BDF}}(E)=\exp \left\{-t_{\mathrm{Be}, \mathrm{coh}}(E) * \rho_{\mathrm{Be}} * \mu_{\mathrm{Be}, \mathrm{coh}}(E)\right\}
$$

Figure 12 shows the best-fit model for the Resolve FM Be window. The best-fit parameters are: $T_{\text {Be,photo+inc }}(E)=252.5 \mu \mathrm{m}$ for Equation 8, values in Table 3 for Equation 9, and those in Table 4 for Equation 10. The difference between the data and the model is smaller than $\sim 0.3 \%$ over a wide energy range, which satisfies the calibration requirements described in Section 1. The mass attenuation coefficients by NIST have some uncertainties. However, they are accounted to some extent by being multiplied by a free parameter. In total, we estimate the transmission uncertainty smaller than $\sim 0.3 \%$ in $2.6-12.0 \mathrm{keV}$.

As shown in Figure 8, the Be thickness has a spatial non-uniformity of $\pm 4.2 \%$. We plan to include this in CALDB as a spatial map. Other spatial differences, such as the absorption edges by the impurities and the BDFs, were found small enough not to affect the observation results based on the comparison of the measurements at three positions (center, top, and bottom) of the Be window. 


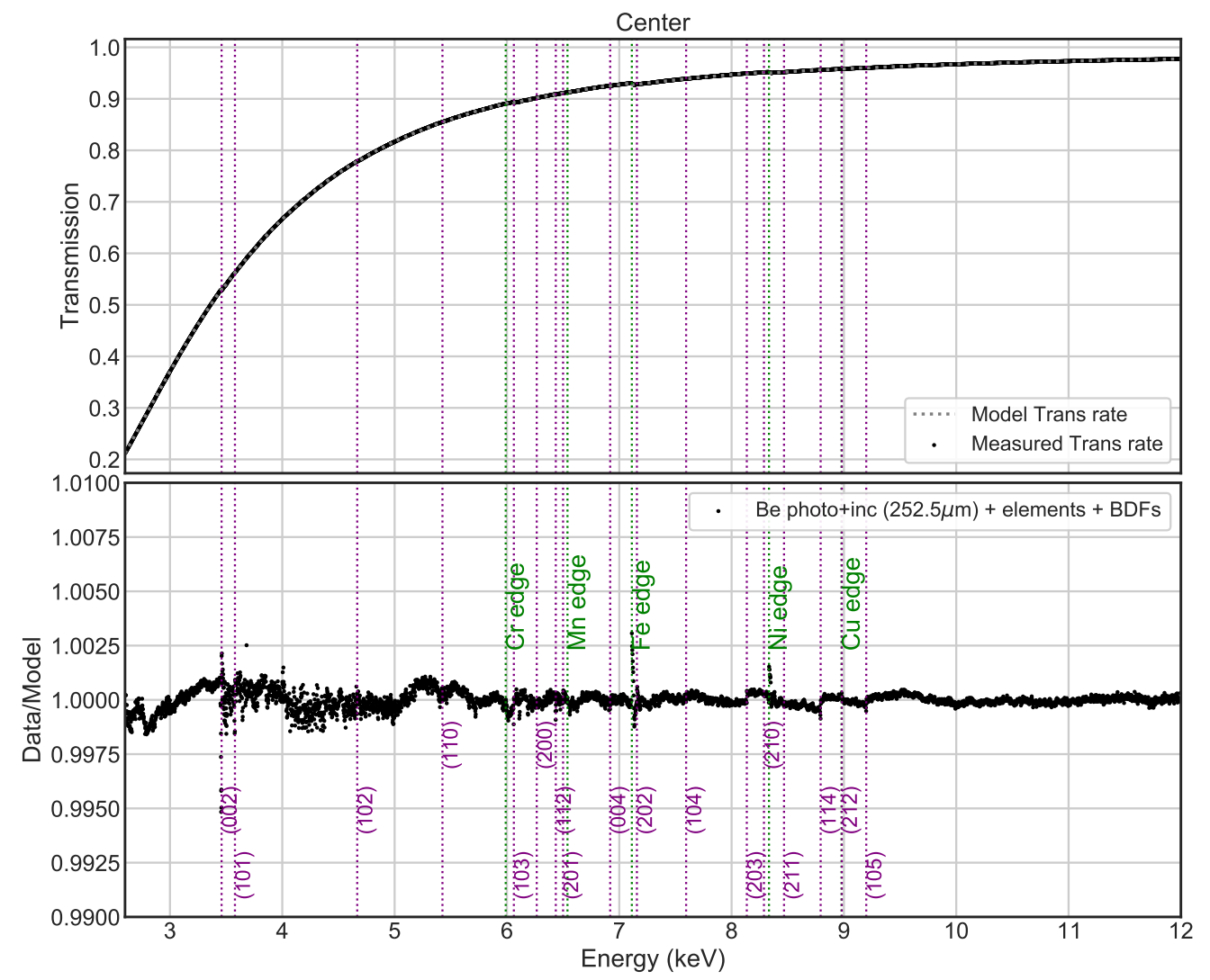

Fig 12 Data and best-fit transmission model of the FM Be window in 2.6-12.0 keV (top). The difference between the data and the model (bottom) is smaller than $\sim 0.2 \%$ in the entire measured energy range. The edges by the contaminants are shown in green, while the BDFs of the labeled Miller index is in purple.

Figure 13 shows the three transmission components expressed in Equation (7). Photoelectric absorption and incoherent scattering of Be (blue) dominates the overall transmission curve. The BDFs (green) and photoelectric absorption of impurities (orange) account for local edge-like features expanded in the inset. 


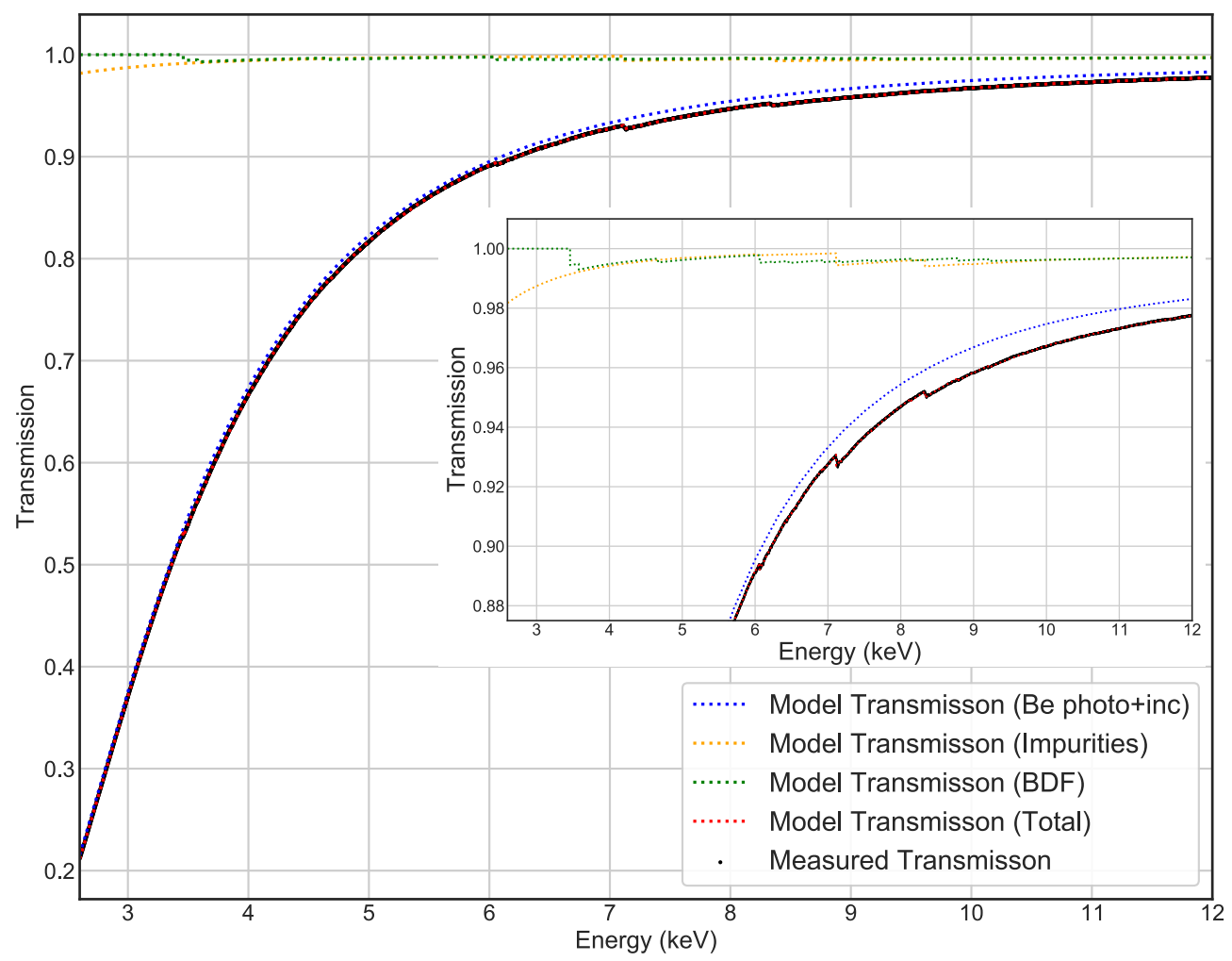

Fig 13 Comparison of the three transmission model components in Equation (7). Inset is an enlarged view of the area where the $y$-axis is greater than 0.875 .

The BDF model in this study is quite similar with that developed for SXS by Yoshida et al. (2017). ${ }^{8}$ Both have step-wise discontinuities followed by a $E^{-2}$ dependency on photon energy $E$.

We gave some physical explanation for this behavior and could impose some constraints global to the energy range. Together with improved data quality, we could better constrain the model parameters now.

\subsection{Modeling Total Transmission of the GV}

We derived the best-fit model for the stainless steel mesh $T_{\text {mesh }}(E)$ in Section 4.1 and the Be window $T_{\mathrm{Be}}(E)$ in Section 4.2. We finally multiply them to obtain the total transmission curve of 
the $\mathrm{GV} T(E)$ as

$$
T(E)=T_{\mathrm{Be}}(E)\left\{f+(1-f) T_{\operatorname{mesh}}(E)\right\},
$$

where $f$ is fixed to the best-fit value of 0.723 . The model was extrapolated to cover the full range from 10 to $40000 \mathrm{eV}$ and interpolated to fill in the $0.25 \mathrm{eV}$ pitch to deliver as a Resolve CALDB product.

The ground calibration result presented here has some limitations, which need to be verified with in-orbit observations. We will (1) evaluate any systematics caused by differences in the measurement setup and actual observation such as beam angles, the detector solid angle, etc, and (2) check the validity of the linear extrapolation of the transmission model out of the measured energy range. We have several free parameters in our model. Fine-tuning these parameters will be achieved by observing continuum sources such as pulsar wind nebulae and blazers in reasonable telescope times before and after the GV open. ${ }^{13,21}$

\subsection{Revisit of Crab Spectrum Obtained with Hitomi SXS}

We have so far discussed the transmission modeling in the Resolve GV. Our approach in the GV modeling can be applied to the ground measurement data taken for the SXS and be compared to the observed data with the SXS in orbit. For the stainless steel mesh, no measurements were done for the SXS, so we fixed the opening fraction $f=0.71$ based on the drawings. This is because, as seen in Figure 9, the mesh is optically thick in the spectral fitting range $2-12 \mathrm{keV}$ and the influence on the residuals is small. For the Be window, we used the ground measurement results of the SXS FS model ${ }^{8}$ obtained in the same setup with Section 3.2 and applied our modeling in Section 4.2. We derived the best-fit Be photoelectric absorption thickness to be $272.5 \mu \mathrm{m}$. 
We generated a Crab spectrum based on the IACHEC model of the Crab nebula established for cross-calibration purposes. ${ }^{22}$ The model is a simple power-law of a photon index 2.10 and a normalization of 8.70 attenuated by an interstellar absorption of an equivalent hydrogen column density of $0.42 \times 10^{22} \mathrm{~cm}^{-2}$. We convolved the model with the redistribution matrix function (RMF) of the SXS CALDB and an ancillary response file (ARF) and compared it to the observed spectrum. We modified the ARFs by allowing some deviations in the Be window thickness. This is because that the FM Be window used in orbit is different from the FS Be window used in the ground measurement and that the difference can be as large as the level of spatial non-uniformity found in the Resolve FM Be window of $\pm 4.2 \%$ (Section 3.3). We made a comparison with the data for the modified ARFs of a Be thicknesses of 261, 266, 271, 276, and $281 \mu \mathrm{m}$ and computed the $\chi^{2}$ values.

Figure 14 shows the comparison between the data and the model in the SXS CALDB (blue) and the best one (261 $\mu \mathrm{m})$ among our deviated thicknesses (red). We confirmed that the best model better describes the Crab observation results. Further improvement is hampered by other sources of calibration uncertainties such as Au M edge features by the X-ray mirrors. 


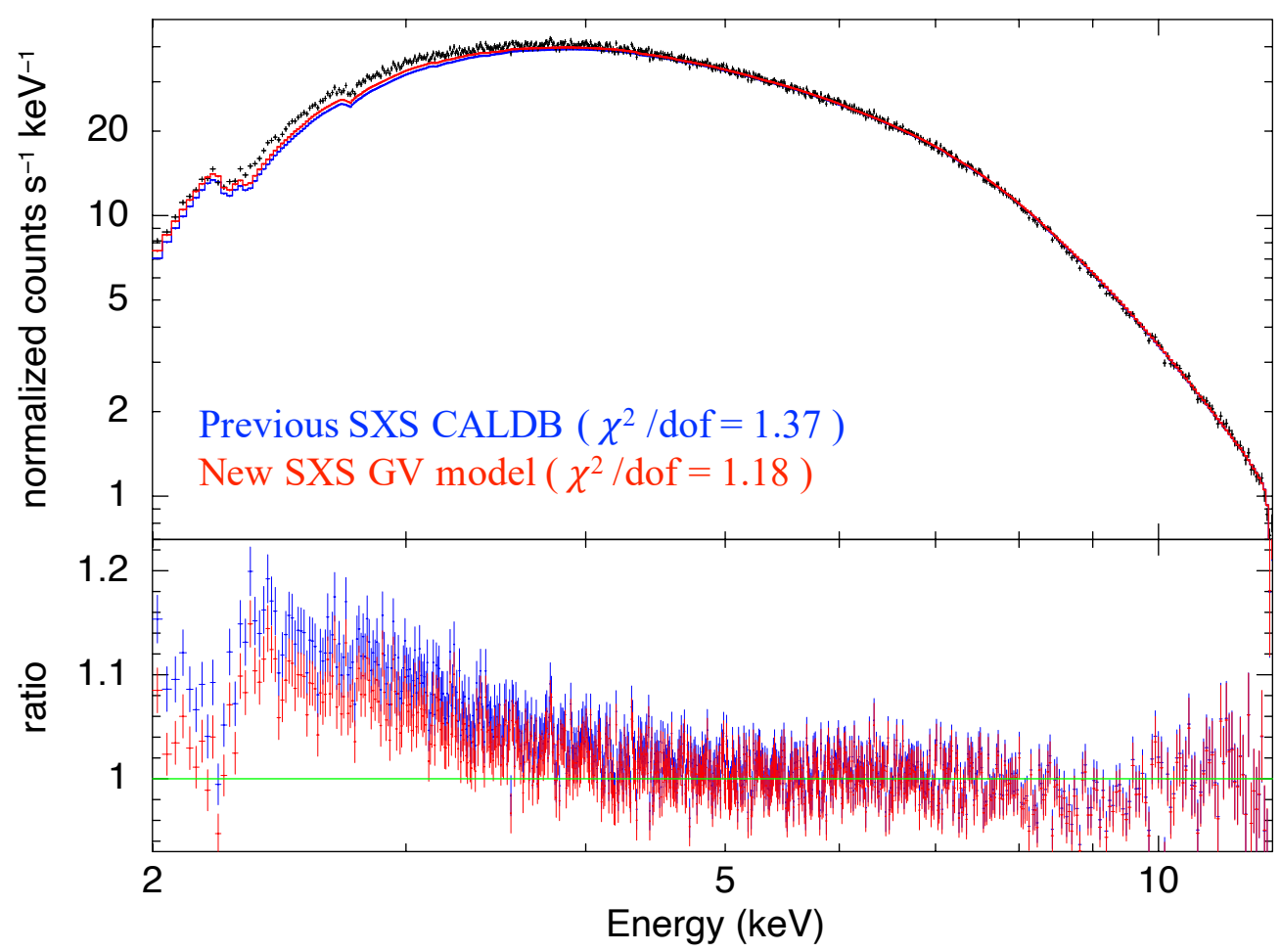

Fig 14 Comparison of simulated Crab spectra with two different ARFs (blue and red) and the observed data with the SXS (black). The blue and red curves respectively show the models using the latest SXS CALDB and the one developed in this study, where the best Be thickness is $261 \mu \mathrm{m}$.

\section{SUMMARY AND CONCLUSION}

We performed the X-ray transmission measurements of the two main components of the Resolve GV: the Be window and the stainless steel mesh. The Be window of the Resolve FM was measured in the $2.0-12.0 \mathrm{keV}$ using the synchrotron facility at KEK PF. The FS stainless steel mesh of the SXS was measured at six discrete energies using the X-ray beamline at ISAS. The stainless steel mesh was measured for the first time in this study.

We developed the X-ray transmission model of the GV. For the stainless steel mesh, the wire cross section was changed from a square in the SXS model to a circle. The mesh opening fraction was derived by fitting the data rather than being fixed to the design value. For the Be window, the following factors were modeled: (1) the photoelectric absorption and incoherent scattering 
of $\mathrm{Be},(2)$ the photoelectric absorption of impurities $(\mathrm{Cr}, \mathrm{Mn}, \mathrm{Fe}, \mathrm{Ni}$, and $\mathrm{Cu}$ ), and (3) the coherent scattering of Be. The physical interpretation of the transmission discontinuity caused by the Bragg diffraction was presented and incorporated into the model. We also measured a spatial non-uniformity in the Be window transmission. All these measurements and models satisfy the calibration requirements.

In addition to constructing the transmission model of the Resolve $\mathrm{GV}$, we revisited the models of the SXS GV based on the approach developed in this work. We performed the reanalysis of the Crab spectrum observed with the SXS when the GV was closed using the ground measurement data and revising the model parameters. We found that the new model better explains the Crab observation results.

In this study, we performed an elaborated X-ray transmission modeling of the Be window considering the beryllium's poly-crystal structure. We also showed utility of measuring X-ray diffraction pattern in addition to the X-ray transmission. We expect that the presented result will serve as a useful reference for all future X-ray spectrometers with a Be window. 


\section{Appendix A: BDF energies}

Table A. 1 summarizes all crystal planes $(h k l)$ of Be below $12.0 \mathrm{keV}$, their spacing $d_{h k l}$, multiplicity $M$, and the BDF energy $E_{\mathrm{BDF}}$ of the $(h k l)$ plane. We also label whether each plane satisfies the systematic absence condition for the Be hexagonal crystal that

$$
-\frac{h}{3}+\frac{k}{3}+\frac{l}{2}=\frac{2 n-1}{2}
$$

where $n$ is an integer. 
Table A.1 Crystal information for crystal planes of Be below $12.0 \mathrm{keV}$

\begin{tabular}{|c|c|c|c|c|}
\hline$h k l$ & $d_{h k l}[\AA]$ & $E_{\mathrm{BDF}}[\mathrm{keV}]$ & $M$ & Systematic absence \\
\hline 001 & 3.5842 & 1.7298 & 2 & absent \\
\hline 100 & 1.9791 & 3.1326 & 6 & \\
\hline 002 & 1.7921 & 3.4596 & 2 & \\
\hline 101 & 1.7325 & 3.5785 & 12 & \\
\hline 102 & 1.3284 & 4.6671 & 12 & \\
\hline 003 & 1.1947 & 5.1894 & 2 & absent \\
\hline 110 & 1.1427 & 5.4259 & 6 & \\
\hline 111 & 1.0887 & 5.6949 & 12 & absent \\
\hline 103 & 1.0228 & 6.0616 & 12 & \\
\hline 200 & 0.9896 & 6.2653 & 6 & \\
\hline 112 & 0.9635 & 6.4350 & 12 & \\
\hline 201 & 0.9539 & 6.4997 & 12 & \\
\hline 004 & 0.8961 & 6.9191 & 2 & \\
\hline 202 & 0.8663 & 7.1570 & 12 & \\
\hline 113 & 0.8258 & 7.5080 & 12 & absent \\
\hline 104 & 0.8163 & 7.5953 & 12 & \\
\hline 203 & 0.7621 & 8.1353 & 12 & \\
\hline 210 & 0.748 & 8.2882 & 12 & \\
\hline 211 & 0.7323 & 8.4668 & 24 & \\
\hline 005 & 0.7168 & 8.6489 & 2 & absent \\
\hline 114 & 0.7051 & 8.7929 & 12 & \\
\hline 212 & 0.6903 & 8.9812 & 24 & \\
\hline 105 & 0.674 & 9.1988 & 12 & \\
\hline 204 & 0.6642 & 9.3342 & 12 & \\
\hline 300 & 0.6597 & 9.3979 & 6 & \\
\hline 301 & 0.6488 & 9.5558 & 12 & absent \\
\hline 213 & 0.634 & 9.7787 & 24 & \\
\hline 302 & 0.6191 & 10.0145 & 12 & \\
\hline 115 & 0.6072 & 10.2100 & 12 & absent \\
\hline 006 & 0.5974 & 10.3787 & 2 & \\
\hline 205 & 0.5805 & 10.6798 & 12 & \\
\hline 303 & 0.5775 & 10.7355 & 12 & absent \\
\hline 214 & 0.5742 & 10.7967 & 24 & \\
\hline 106 & 0.5719 & 10.8412 & 12 & \\
\hline 220 & 0.5713 & 10.8518 & 6 & \\
\hline 221 & 0.5642 & 10.9888 & 12 & absent \\
\hline 310 & 0.5489 & 11.2949 & 12 & \\
\hline 222 & 0.5443 & 11.3899 & 12 & \\
\hline 311 & 0.5426 & 11.4266 & 24 & \\
\hline 304 & 0.5313 & 11.6703 & 12 & \\
\hline 116 & 0.5294 & 11.7114 & 12 & \\
\hline 312 & 0.5248 & 11.8128 & 24 & \\
\hline 215 & 0.5176 & 11.9791 & 24 & \\
\hline
\end{tabular}




\section{Acknowledgments}

The authors acknowledge Megan E. Eckart (LLNL), Maurice A. Leutenegger (NASA GSFC), Yoh Takei (JAXA), and anonymous reviewers for their valuable comments. This work was performed under the approval of the Photon Factory Program Advisory Committee (Proposal No. 2018G509) and Proposal Assessing Committee of HiSOR (Proposal No. 19BU007). We acknowledge Shinjiro Hayakawa (Hiroshima University) for the upgrading the HiSOR BL-11 beamline and supporting our measurements. We appreciate Koichi Kitazono and Yuta Fujimori (Tokyo Metropolitan University) for allowing us to use their X-ray diffractometer and supporting our experiments. We used software, API, and databases provided by the Cambridge Crystallographic Data Centre. T.M is financially supported by JSPS Grant-in-Aid for JSPS Research Fellow (JP20J20809). A part of the results of this manuscript was previously reported in SPIE proceedings. ${ }^{23}$

\section{References}

1 M. Tashiro, H. Maejima, K. Toda, et al., "Status of x-ray imaging and spectroscopy mission (XRISM)," Proc. SPIE 11444, 1144422 (2020).

2 R. L. Kelley, H. Akamatsu, P. Azzarello, et al., "The Astro-H high resolution soft x-ray spectrometer," Proc. SPIE 9905, 99050V (2016).

3 T. Takahashi, M. Kokubun, K. Mitsuda, et al., "Hitomi (ASTRO-H) X-ray Astronomy Satellite,” Journal of Astronomical Telescopes, Instruments, and Systems 4(2), 021402 (2018).

4 Y. Ishisaki, Y. Ezoe, S. Yamada, et al., "Resolve Instrument on X-ray Astronomy Recovery Mission (XARM),” Journal of Low Temperature Physics 193, 991-995 (2018).

5 R. Fujimoto, Y. Takei, K. Mitsuda, et al., "Performance of the helium dewar and the cryocool- 
ers of the Hitomi soft x-ray spectrometer," Journal of Astronomical Telescopes, Instruments, and Systems 4, 011208 (2017).

6 T. Ohashi, K. Ebisawa, Y. Fukazawa, et al., "The Gas Imaging Spectrometer on Board ASCA," Publications of the Astronomical Society of Japan 48, 157-170 (1996).

7 D. Barret, T. Lam Trong, J.-W. den Herder, et al., "The ATHENA X-ray Integral Field Unit (X-IFU)," Proc. SPIE 10699, $106991 \mathrm{G}$ (2018).

8 Y. Yoshida, J. Sugimoto, R. Ishii, et al., "Transmission measurement of the spare Beryllium window of the SXS onboard the Hitomi satellite in 2.0-12 keV with KEK-PF," Proc. SPIE 10397, 103971D (2017).

9 M. E. Eckart, J. S. Adams, K. R. Boyce, et al., "Ground calibration of the Astro-H (Hitomi) soft x-ray spectrometer," Journal of Astronomical Telescopes, Instruments, and Systems 4(2), 021406 (2018).

10 A. Hoshino, Y. Yoshida, S. Kitamoto, et al., "The evaluation of the Hitomi (Astro-H)/SXS spare beryllium window in 3.8-30 keV," Proc. SPIE 10397, 103970E (2017).

11 M. Tsujimoto, T. Okajima, M. E. Eckart, et al., "In-flight calibration of Hitomi Soft X-ray Spectrometer. (3) Effective area," Publications of the Astronomical Society of Japan 70, 20 (2018).

12 Eckart, M. E., Leutenegger, M. A., Okajima, T. et al., "XARM Resolve Calibration Requirements (RESOLVE-SYS-REQ-0017),” (2018).

13 T. Midooka, "Ground calibration of XRISM for the initial observations: X-ray transmission of the gate valve of the cryostat for the low-temperature detector (in Japanese; 2020)," Master's thesis, University of Tokyo (2020). 
14 T. Hayashi, T. Sato, N. Kikuchi, et al., "Upgrade of the 30-m x-ray pencil beam line at the Institute of Space and Astronautical Science," Journal of Astronomical Telescopes, Instruments, and Systems 1(4), 044004 (2015).

15 http://pfwww.kek.jp/users_info/station_spec/bl7/bl7c.html. In Japanese, (Last accessed: 2021.3.19).

16 http://pfwww.kek.jp/sxspec/sx/bl11b_e.html. (Last accessed: 2021.3.19).

17 http://www.hsrc.hiroshima-u.ac.jp/english/storagering_ beamlines/beamlines . html. (Last accessed: 2021.3.19).

18 https://physics.nist.gov/PhysRefData/Xcom/html/xcoml.html. (Last accessed: 2021.3.19).

19 A. Authier, "Optical properties of X-rays - dynamical diffraction," Zeitschrift für Kristallographie 227, 36-51 (2012).

20 https://www2.physics.ox.ac.uk/sites/default/files/ CrystalStructure_fullnotes6.pdf. (Last accessed: 2021.3.19).

21 E. D. Miller, M. Sawada, M. Guainazzi, et al., "Planning in-flight calibration for XRISM," Proc. SPIE 11444, 1144426 (2020).

22 M. C. Weisskopf, M. Guainazzi, K. Jahoda, et al., “ON CALIBRATIONS USING THE CRAB NEBULA AND MODELS OF THE NEBULAR X-RAY EMISSION," The Astrophysical Journal 713, 912-919 (2010).

23 T. Midooka, M. Tsujimoto, S. Kitamoto, et al., "X-ray transmission measurements of the gate valve for the x-ray astronomy satellite XRISM," Proc. SPIE 11444, 114445C (2020). 
First Author is a PhD candidate at the University of Tokyo. He received his BS degree in physics from Kyoto University in 2018, and his MS degree in astronomy from the University of Tokyo in 2020. His current research interests include X-ray and gravitational wave astronomy.

Biographies and photographs of the other authors are not available.

\section{List of Figures}

1 Resolve FM GV at the top of the Dewar

2 Picture of the Be window and the stainless steel mesh

3 Schematic of the ISAS beamline

4 Interior of the measuring chamber in the beamline

5 Setup for measurements at the BL-11B and BL-7C

6 The photo of the BL-11B

7 Transmission curve in $1.8-12.0 \mathrm{keV}$

8 Spatial non-uniformity maps of the Be window transmission

9 Comparison of three transmission models for the stainless steel mesh

10 Results of the X-ray diffraction measurements and fitting

11 Best-fit coherent scattering thickness of the Be with step-wise changes

12 Data and best-fit transmission model of the FM Be window in $2.6-12.0 \mathrm{keV}$

13 Decomposition of the three transmission model components

14 Comparison of simulated Crab spectra with two different ARFs and the observed data with the SXS 


\section{List of Tables}

1 Calibration requirements of the Be window and the stainless steel mesh ${ }^{12}$

2 Transmission measurement results of the stainless steel mesh

3 Areal density of impurities of the FM Be window

$4 \quad$ Fitting results of the coherent scattering thickness of the Resolve FM Be window

A.1 Crystal information for crystal planes of Be below $12.0 \mathrm{keV}$ 